\title{
ABSENTEÍSMO DE TRABALHADORES DE ENFERMAGEM EM UM HOSPITAL UNIVERSITÁRIO
}

\author{
Dóris Marli Petry Paulo da Silva* \\ Maria Helena Palucci Marziale**
}

SILVA, D.M.P.P.da; MARZIALE, M.H.P. Absenteísmo de trabalhadores de enfermagem em um hospital universitário.

Rev.latino-am.enfermagem, Ribeirão Preto, v. 8, n. 5, p. 44-51, outubro 2000.

Objetivando identificar os indice de absenteísmo-doença entre os trabalhadores de enfermagem do hospital universitário, foi realizado um levantamento das faltas por licença-saúde registradas durante doze meses e calculado o indice de freqüencia e a porcentagem de tempo perdido. Os dados foram coletados através de consulta documental aos registros do departamento pessoal da instituição estudada e transcritos em um protocolo. Diante dos resultados obtidos, constatou-se que o maior índice de freqüencia ocorreu na Pediatria $(I f=0,35)$ e a porcentagem de tempo perdido de trabalho ocorreu no Pronto Atendimento $(T p=4,19)$. Concluiu-se que os indices de absenteísmo-doença entre os trabalhadores apresentam-se elevados, indicando a necessidade de criação de um banco de dados para otimizar o registro das faltas, facilitar seu controle e possibilitar futuras pesquisas.

UNITERMOS: absenteísmo, doença, trabalhadores, enfermagem, recursos humanos de enfermagem no hospital

\section{INTRODUÇÃO}

FERREIRA (1986) reportando-se a definição da palavra absenteísmo define-a como ausência habitual do emprego.

O absenteísmo, absentismo ou ausentismo é uma expressão utilizada para designar a falta do empregado ao trabalho. Isto é, a soma dos períodos em que os empregados de determinada organização se encontram ausentes do trabalho, não sendo a ausência motivada por desemprego, doença prolongada ou licença legal (CHIAVENATO, 1994, p.119).

Segundo QUICK \& LAPERTOSA (1982), o absenteísmo é dividido em absenteísmo voluntário (ausência no trabalho por razões particulares não justificada por doença); absenteísmo por doença (inclui todas as ausências por doença ou por procedimento médico, excetuam-se os infortúnios profissionais); absenteísmo por patologia profissional (ausências por acidentes de trabalho ou doença profissional); absenteísmo legal (faltas no serviço amparadas por leis, tais como: gestação, nojo, gala, doação de sangue e serviço militar) e absenteísmo compulsório (impedimento ao trabalho devido a suspensão imposta pelo patrão, por prisão ou outro impedimento que não permita o trabalhador chegar ao local de trabalho).

Para COUTO (1987), o absenteísmo é decorrente de um ou mais fatores causas, tais como, fatores de trabalho, sociais, fatores culturais, de personalidade e de doenças. O referido autor alerta que não parece existir uma relação precisa de causa e efeito, mas sim, um conjunto de variáveis podem levar ao absenteísmo.

O absenteísmo no trabalho abrange todas as causas de ausência, como: doença prolongada, acidentes, licenças de maternidade, atenção a problemas familiares ou formalidades judiciais e até cursos fora da empresa, exceto greve, cursos dentro da empresa, repouso semanal ou compensado, férias e feriados (BULHÕES, 1986).

Segundo OTERO (1993), a etiologia do absenteísmo é multifatorial, dependendo da sua origem. Podem ser classificados em fatores dependentes da atividade laboral, perilaborais, do meio extralaboral, patologias sofridas pelo trabalhador, fatores individuais e fatores dependentes do sistema administrativo.

Um aspecto importante a ser considerado é que as causas do absenteísmo nem sempre estão no trabalhador, mas na empresa, enquanto organização e supervisão deficientes, através da repetitividade de tarefas, da desmotivação e desistímulo, das condições desfavoráveis de ambiente e de trabalho, da precária

\footnotetext{
* Enfermeira do Hospital Universitário da Universidade Estadual de Maringá, mestranda pelo Departamento de Enfermagem Geral e Especializada da Escola de Enfermagem de Ribeirão Preto da Universidade de São Paulo

** Enfermeira, Professor Doutor do Departamento de Enfermagem Geral e Especializada da Escola de Enfermagem de Ribeirão Preto da Universidade de São Paulo
} 
integração entre os empregados e a organização e dos impactos psicológicos de uma direção deficiente, que não visa uma política prevencionista e humanista (ALEXANDRE, 1987; COUTO, 1987; CHIAVENATO, 1994; ALVES, 1995).

Segundo BULHÕES (1998), o absenteísmo pode refletir tanto as condições de saúde do trabalhador, quanto as condições de vida e trabalho, os quais devem ser analisados e prevenidos.

O índice de absenteísmo, segundo CHIAVENATO (1994, p. 172), deve refletir a porcentagem do tempo não trabalhado em decorrência das ausências em relação ao volume de atividade esperada ou planejada.

O número insuficiente de recursos humanos pode contribuir para elevar o índice de absenteísmo, como conseqüência da sobrecarga e insatisfação dos trabalhadores, desencadeando a queda da qualidade do cuidado prestado ao homem (ALVES, 1995).

O absenteísmo na enfermagem é preocupante, pois desorganiza o serviço, gera insatisfação e sobrecarga entre os trabalhadores presentes e conseqüentemente diminui a qualidade da assistência prestada ao paciente.

Segundo REIS (1986), o absenteísmo apresentase como um obstáculo para as chefias de enfermagem manterem a qualidade da assistência, acrescido às limitações no desempenho de suas funções frente aos trabalhos que dispensam um esforço físico maior.

Concordamos com ROBAZZI et al. (1990), que as ausências de funcionários de enfermagem desorganizam o trabalho de equipe e vão alterar a qualidade e quantidade do atendimento prestado ao paciente, desencadeando problemas também aos enfermeiros que ocupam cargos de chefia, pois têm sob sua responsabilidade a resolução de todos os problemas administrativos que lá acontecem.

O absenteísmo pode estar diretamente relacionado às condições de trabalho, refletindo na qualidade e produtividade laboral e na vida do trabalhador de enfermagem.

LOPES et al. (1996) estudando as condições de trabalho e vida dos trabalhadores de enfermagem, observaram que estes estão sendo colocados à mercê de riscos provenientes de condições precárias de trabalho, aos quais são responsáveis pelo aparecimento de doenças.

Para MARZIALE (1995), os trabalhadores de enfermagem estão constantemente expostos aos riscos ocupacionais, relacionados aos agentes físicos, químicos e biológicos e a fatores ergonômicos e psicossociais.

Segundo GASPAR (1997), os riscos para a saúde relacionados com o trabalho dependem do tipo de atividade profissional e das condições em que a mesma é desempenhada. $\mathrm{O}$ autor complementa, os serviços de saúde, em particular, os hospitais, proporcionam aos seus trabalhadores condições de trabalho reconhecidamente piores em relação aos demais serviços de saúde.

As condições de trabalho da enfermagem implicam em longas jornadas, no trabalho em turnos desgastantes (vespertinos e noturno, domingos e feriados), nos rodízios, em multiplicidade de funções, repetitividade e monotonia, intensividade e ritmo excessivo de trabalho, ansiedade, esforços físicos, posições incômodas, na separação do trabalho intelectual e manual, no controle das chefias, desencadeando acidentes e doenças (ALVES,1995).

Muitas vezes, trabalhadores com problemas de saúde, quer físicos e/ou mentais, segundo MOURA (1992) transferem seus problemas para o trabalho, os quais podem ocasionar atrasos, faltas, descuido com o material e queda na qualidade do trabalho executado.

Os riscos ocupacionais variam de acordo com as atividades exercidas e o meio ambiente. A sobrecarga de risco pode desencadear prejuízo para a saúde do trabalhador, provocando o absenteísmo.

Considerando que, as faltas ao trabalho acarretam problemas na organização do trabalho, prejudicam a assistência de enfermagem prestada aos pacientes e indicam a existência de problemas preocupantes quando relacionados às condições de saúde, os autores sentiramse motivados a realizar o estudo ora apresentado.

\section{OBJETIVOS}

\section{Objetivo Geral}

Investigar o absenteísmo por doença junto aos trabalhadores de enfermagem de um hospital universitário.

\section{Objetivos Específicos}

Levantar a incidência do absenteísmo por doença junto aos trabalhadores de enfermagem de um hospital universitário.

Calcular o tempo perdido de trabalho ocasionado por problemas de saúde nos trabalhadores de enfermagem.

\section{METODOLOGIA}

Trata-se de uma pesquisa embasada na abordagem positivista, com análise quantitativa dos dados observando-se a padronização do Subcomitê de Absenteísmo da Associação Internacional de Medicina do Trabalho, para os cálculos dos índices de freqüência e da porcentagem de tempo perdido (COUTO, 1987).

Foi realizado um levantamento retrospectivo sobre a incidência de absenteísmo e suas causas, no período de um ano, ocorridos entre os trabalhadores de 
enfermagem de uma instituição hospitalar.

As fórmulas utilizadas para os cálculos de Índice de Freqüência(If) e Porcentagem de Tempo Perdido (Tp) encontram-se descritas à seguir:

$$
\text { Índice de freqüência } \quad=\quad \frac{\text { Número de licenças no período }}{\text { Efetivo médio do período }}
$$

Porcentagem de $\quad=$

Tempo Perdido

\section{Local}

Hospital Universitário do interior do Paraná.

\section{População}

Considerou-se como universo deste estudo a equipe de enfermagem pertencente à Diretoria de Enfermagem da instituição, no período de 01/07/97 à 30/ 06/98, perfazendo um total de 199 trabalhadores, sendo 47 enfermeiros e 152 auxiliares de enfermagem.

\section{Procedimentos}

Inicialmente foi encaminhado uma solicitação à Comissão de Ética do Hospital para a realização da pesquisa. De posse do parecer favorável foram coletados os dados junto ao registro do departamento pessoal do hospital estudado, através de um protocolo de coleta de dados elaborado pelos autores.

Os dados foram ordenados através do Programa Microsoft Exce1/97. Para a análise dos resultados, foram utilizados métodos descritivos, agrupando-se as respostas em tabelas (distribuições de freqüência) e gráficos.

\section{RESULTADOS E DISCUSSÃO}

Primeiramente apresentamos algumas características da população estudada. Os trabalhadores de enfermagem participantes do estudo, possuíam tempo de serviço superior a 2 anos $(58,8 \%)$, idade inferior a 40 anos(79,8\%), predomínio do sexo feminino $(89,9 \%)$, estado civil casado (52,3\%) e com filhos $(68,3 \%)$.

Através do levantamento do absenteísmo-doença entre os 199 trabalhadores de enfermagem, ocorrido no período estudado, constatamos que 26 trabalhadores (13\%) não apresentaram faltas durante o período estudado. No entanto, 173 trabalhadores (87\%) ausentaram-se do serviço; desses, 150 (75\%) justificaram sua ausência por motivo de doenças e os demais por outros motivos, incluindo os acidentes de trabalho.

A Figura 1 mostra a distribuição dos afastamentos segundo os motivos apresentados.

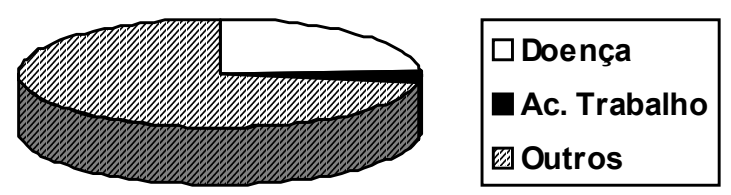

Figura 1 - Gráfico da distribuição dos trabalhadores participantes do estudo, segundo motivo dos afastamentos

Neste estudo foi considerado, absenteísmo por doença (72,6\%) a ausência do trabalhador justificada por atestado ou laudo médico, que representa 494 ausências com 1.491 dias perdidos de trabalho, no período de um ano.

Segundo OTERO (1993), a maioria das ausências são justificadas por atestados médicos, o que não significa que todas as ausências sejam sempre decorrentes de causas médicas.

Absenteísmo por acidente de trabalho (2,9\%), como sendo toda ausência decorrente de algum acidente sofrido durante a jornada de trabalho ou de percurso, equivalentes a 20 afastamentos com 305 dias perdidos.

Segundo NAPOLEÃO (1999), os acidentes de trabalho na enfermagem são subnotificados, sobretudo, devido à falta de conhecimento sobre o que é o acidente, de trabalho, falta de tempo para notificá-lo, burocracia e medo de perder o emprego, por considerar a ocorrência do acidente de trabalho como uma culpa.

Em relação ao absenteísmo por outros motivos $24,5 \%$ das faltas ao trabalho justificadas por atestados ocorreram devido a necessidade de acompanhamento de familiares a tratamento de saúde, doação de sangue, licença nojo e gala, dispensa para audiências e liberação para participação em eventos/treinamentos representados por 166 faltas com perda de 413 dias.

Para JORGE (1995, p.40), "as questões de saúde familiar tem gerado absenteísmo na equipe de enfermagem". 
Os resultados apresentados na Figura 1 confirmam o estudo de LOPES et al. (1996), quando analisou o comportamento das ausências não previstas por licenças e faltas em trabalhadores de enfermagem, onde as licenças médicas correspondem a $75,5 \%$ das ausências a as faltas por outros motivos, à $24,5 \%$.

Além das faltas dos trabalhadores ao serviço, existe legalmente o afastamento ao trabalho por direito adquirido do trabalhador, como: férias, abonos, licença prêmio e licença maternidade (120 dias), sem prejuízo de seus proventos salariais. Férias e folgas são consideradas ausências planejadas em escala dentro de uma jornada de trabalho legal, não prejudicando a qualidade da assistência prestada. Estes afastamentos legais não foram computados como ausências em nosso estudo.

CHIAVENATO (1985) considera como causas do absenteísmo: doenças efetivamente comprovadas; doenças não comprovadas; razões diversas de caráter familiar; atrasos involuntários; e faltas voluntárias por motivos diversos. Segundo o autor, não se deve incluir o acidente de trabalho entre as causas do absenteísmo, quando se pretende comparar os índices de absenteísmo.

Através da Tabela 1 é apresentada a distribuição do número de afastamentos, segundo categoria profissional e motivos do afastamento:

Tabela 1 - Distribuição dos trabalhadores de enfermagem participantes do estudo, segundo categoria profissional e motivo do afastamento: número de afastamentos e dias perdidos de trabalho.

\begin{tabular}{l|cc|cccc|cc|cc}
\hline \multirow{2}{*}{$\begin{array}{l}\text { Categoria } \\
\text { Profissional }\end{array}$} & \multicolumn{7}{|c|}{ Motivo do Afastamento } \\
\cline { 2 - 11 } & \multicolumn{2}{|c|}{ Doença } & Acidente & De & Trabalho & \multicolumn{2}{c}{ Outros } & \multicolumn{2}{c}{ Total } \\
\hline \multirow{2}{*}{$\mathrm{A.E}^{*}$} & 419 & 1212 & 14 & 20 & 305 & 121 & 290 & 560 & 1807 \\
\hline ENF** & 75 & 279 & - & - & - & 45 & 123 & 120 & 402 \\
\hline TOTAL & 494 & 1491 & 14 & 20 & 305 & 166 & 413 & $\mathbf{6 8 0}$ & $\mathbf{2 2 0 9}$ \\
\hline
\end{tabular}

* Auxiliar de Enfermagem

** Enfermeiro

Constatamos através da Tabela 1 que, dos 560 afastamentos apresentados pelos auxiliares de enfermagem, 419 (74,7\%) foram justificados por doença, sendo, responsáveis por 1.212 dias perdidos de trabalho, 20 afastamentos $(3,6 \%)$ por acidentes de trabalho, com perda de 305 dias de trabalho e 121 faltas $(21,7 \%)$ por outros motivos, acarretando 290 dias ausentes ao trabalho. Houve ainda o registro de 14 acidentes de trabalho sem afastamentos.

Os setores dos trabalhadores onde se registraram os maiores números de ausência no trabalho por doença, foram o Centro de Material, onde 91,6\% dos trabalhadores apresentaram pelo menos um afastamento por doença durante o período estudado, seguido do Centro Cirúrgico e da Gineco-Obstetrícia com 84,6\% respectivamente.

Os locais de trabalho que registraram os maiores números de dias perdido foram o Pronto Atendimento com 301 dias perdidos $(24,8 \%)$, seguido da Pediatria com 197 dias perdidos (16,3\%), Gineco-Obstetrícia com 117 dias perdidos $(9,6 \%)$ e da Clínica Médica com 110 dias perdidos $(9,1 \%)$.

Os enfermeiros apresentaram 120 afastamentos do trabalho, sendo 75 faltas ocasionadas por doença (62,5\%), representando 279 dias perdidos de trabalho, e
45 faltas justificadas por outros motivos $(37,5 \%)$, com 123 dias perdidos. Ressaltamos que não houve registro de acidentes de trabalho entre os enfermeiros; este dado nos traz a indagação em relação a subnotificação de acidentes de trabalho.

Quanto ao local de trabalho, os enfermeiros das UTIs ausentaram-se mais devido a doenças $(82,6 \%)$ em relação aos outros setores, apresentando maior número de dias perdidos por doença $(66,7 \%)$ ou seja 186 dias.

Em relação ao tempo perdido de trabalho decorrente dos afastamentos registrados no período estudado, podemos observar através da Tabela 1 que os 680 afastamentos proporcionaram 2.209 dias perdidos. Os trabalhadores de enfermagem tiveram 1.491 dias perdidos por motivo de doença, 305 dias perdidos com acidentes de trabalho e 413 dias perdidos por outros motivos.

As duas categorias profissionais apresentaram número de faltas proporcionais. (Auxiliar de Enfermagem $=74,8 \%$ e Enfermeiro $=62,5 \%$ ).

Em relação à incidência do absenteísmo por doença, os dados são descritos a seguir.

Na Tabela 2 são apresentados os dados relativos aos atestados e laudos médicos e número de dias afastados do trabalho. 
Tabela 2 - Distribuição dos trabalhadores adoecidos participantes do estudo, segundo tipo de atestados e número de dias afastados do trabalho

\begin{tabular}{l|cccc}
\hline \multirow{2}{*}{ Tipo de atestados } & \multicolumn{4}{|c}{ Número de dias afastados do trabalho } \\
\cline { 2 - 5 } & $\mathbf{1}$ a 2 dias & $\begin{array}{c}\mathbf{3} \text { a } \mathbf{~ 1 4} \\
\text { dias }\end{array}$ & $\begin{array}{c}\mathbf{1 5} \text { ou } \\
\text { mais dias }\end{array}$ & Total \\
\hline Atestados com CID & 292 & 26 & 23 & 341 \\
Atestados sem CID & 99 & 33 & 21 & 153 \\
\hline Total & $\mathbf{3 9 1}$ & $\mathbf{5 9}$ & $\mathbf{4 4}$ & $\mathbf{4 9 4}$ \\
\hline
\end{tabular}

Analisando as justificativas dos afastamentos, constatamos que, em 150 trabalhadores de enfermagem afastados por doença no período estudado, foram registrados 494 atestados ou laudos médicos, dos quais 341 atestados continham o diagnóstico descrito e 153 atestados não continham diagnóstico. Cabe ressaltar que alguns atestados não contém o diagnóstico ou código da Classificação Internacional de Doenças (CID), alegando como um direito do trabalhador, para não ser exposto.

Observamos, através da Tabela 2, o predomínio de atestados por 1 ou 2 dias de dispensa ao trabalho. Este fato é justificado, uma vez que atestados de 1 a 2 dias dispensam perícia médica e à partir do $3^{\circ}$ dia, o trabalhador deve se apresentar para perícia médica junto ao Instituto de Previdência do Estado (IPE), sendo então fornecido o laudo médico. O laudo médico normalmente não contém o diagnóstico. Este procedimento é válido para todo trabalhador de instituição pública.

A categorização de 1 a 2 dias, 3 a 14 dias e 15 ou mais dias, deve-se ao fato de que em instituições privadas, a empresa arca com o ônus do trabalhador afastado por doença e acima de 15 dias, o trabalhador fica afastado com seus benefícios pagos pelo governo.

BITTENCOURT (1993), observou a ocorrência de tempo de afastamento do trabalho por doença em trabalhadores de enfermagem, sendo que $74,2 \%$ afastaram-se até 15 dias e $25,8 \%$ permaneceram ausentes de suas atividades por um período superior a 15 dias.

MEIRA (1982) constatou que mais de $80 \%$ de todas as ausências tem duração igual ou inferior a três dias, contribuindo com menos de $15 \%$ dos dias perdidos; e que menos de $10 \%$ dos casos são responsáveis por mais de $80 \%$ dos dias perdidos.

Apresentamos, através da Tabela 3, os resultados dos cálculos do Índice de Freqüência (If) e de Porcentagem de Tempo Perdido (\%Tp) acumulados durante doze meses, isto é, de 1 de julho de 1997 à 30 de junho de 1998.

Tabela 3 - Distribuição dos trabalhadores participantes do estudo segundo categoria profissional e local de trabalho e: número de afastamentos por doença, índice de freqüência, número de dias perdidos e tempo perdido de trabalho

\begin{tabular}{|c|c|c|c|c|c|c|}
\hline \multicolumn{2}{|l|}{$\begin{array}{c}\text { Categoria } \\
\text { Profissional }\end{array}$} & \multirow{2}{*}{$\begin{array}{l}\text { Local de Trabalho } \\
\text { UTI }\end{array}$} & \multirow{2}{*}{$\begin{array}{c}\begin{array}{c}\text { Número de } \\
\text { Afasta/os } \\
\text { Por Doença }\end{array} \\
34\end{array}$} & \multirow{2}{*}{$\begin{array}{c}\begin{array}{c}\text { Indice de } \\
\text { Frequiência } \\
\text { (If) }\end{array} \\
0,19\end{array}$} & \multirow{2}{*}{$\begin{array}{c}\begin{array}{c}\text { Número de } \\
\text { Dias Perdidos } \\
\text { de Trabalho }\end{array} \\
85\end{array}$} & \multirow{2}{*}{$\begin{array}{c}\begin{array}{c}\text { Porcentagem } \\
\text { de Tempo } \\
\text { Perdido (Tp) }\end{array} \\
1,88\end{array}$} \\
\hline \multirow{3}{*}{$\begin{array}{l}\text { Auxiliar de } \\
\text { Enfermagem }\end{array}$} & UTI & & & & & \\
\hline & DI & $\begin{array}{l}\text { Banco de leite } \\
\text { Berçário } \\
\text { Pediatria } \\
\text { Gineco Obstetrícia } \\
\text { Clínica Médica } \\
\text { Clínica Cirúrgica }\end{array}$ & $\begin{array}{l}03 \\
32 \\
62 \\
38 \\
49 \\
34\end{array}$ & $\begin{array}{l}0,25 \\
0,25 \\
0,35 \\
0,23 \\
0,26 \\
0,17\end{array}$ & $\begin{array}{l}05 \\
89 \\
197 \\
117 \\
110 \\
94\end{array}$ & $\begin{array}{l}0,01 \\
3,08 \\
4,14 \\
2,89 \\
2,36 \\
1,85\end{array}$ \\
\hline & DA & $\begin{array}{l}\text { Ambulatório } \\
\text { Pronto Atendimento } \\
\text { Centro Cirúrgico } \\
\text { Centro de Material }\end{array}$ & $\begin{array}{l}27 \\
71 \\
29 \\
40\end{array}$ & $\begin{array}{l}0,24 \\
0,25 \\
0,18 \\
0,27\end{array}$ & $\begin{array}{c}77 \\
301 \\
41 \\
96\end{array}$ & $\begin{array}{l}2,81 \\
4,19 \\
1,02 \\
2,66\end{array}$ \\
\hline \multirow[b]{2}{*}{ Enfermeiro } & Total & & 419 & 2,64 & 1212 & 26,89 \\
\hline & $\begin{array}{l}\text { UTI } \\
\text { DI } \\
\text { DA }\end{array}$ & $\begin{array}{l}\text { UTI } \\
\text { Ber,Ped,GO,Cir,Med } \\
\text { Amb,PA,CCir,CM at }\end{array}$ & $\begin{array}{l}31 \\
26 \\
18\end{array}$ & $\begin{array}{l}0,16 \\
0,14 \\
0,10\end{array}$ & $\begin{array}{l}186 \\
54 \\
39\end{array}$ & $\begin{array}{l}4,14 \\
1,12 \\
1,00\end{array}$ \\
\hline Sub & Total & & 75 & 0,4 & 279 & 6,26 \\
\hline \multicolumn{3}{|c|}{ TOTAL } & 494 & 3,04 & 1491 & 33,15 \\
\hline
\end{tabular}


Através da Tabela 3 observamos que, durante o período estudado, foram registrados 494 afastamentos, correspondendo a 1.491 dias de trabalho perdidos pelos trabalhadores adoecidos.

Entre os auxiliares de enfermagem, aqueles lotados na Pediatria foram os que apresentaram o maior índice de freqüência, isto é, If igual a 0,35 equivalente a 62 afastamentos, seguidos dos lotados no Centro de Material (If $=0,27$ com 40 afastamentos) e dos lotados no Pronto Atendimento ( 71 afastamentos e If $=0,25$ ). Em relação à porcentagem de tempo perdido, observamos que os trabalhadores adoecidos do Pronto Atendimento apresentaram 4,19\% de tempo perdido com doença, ou seja, 301 dias perdidos e da Pediatria ausentaram-se $4,14 \%$ do tempo, equivalentes a 197 dias perdidos de trabalho.

Os enfermeiros da UTI ausentaram-se mais por doença em relação as outras causas e apresentaram 186 dias perdidos com $4,14 \%$ do tempo perdido.

Segundo COUTO (1987), para a realidade brasileira, atualmente, podemos classificar como índices de absenteísmo-doença excessivos, com valores de índice de freqüência (If) maiores que 0,10 por mês. Isto é, num determinado mês, para cada 100 trabalhadores ocorreram mais de 10 afastamentos por doença. $\mathrm{O}$ autor considera a porcentagem de tempo perdido (\% $\%$ p) acumulado maior que $1,2 \%$ alto, recomendando que a situação do trabalho deve ser avaliada.

Observamos que o Índice de freqüência entre os auxiliares de enfermagem da instituição em estudo apresenta-se elevado na maioria dos setores, e entre os enfermeiros também está elevado em determinados meses.

MARZIALE (1995), investigando o absenteísmo-doença em trabalhadores de enfermagem, constatou que a unidade de Pediatria Cirúrgica apresentava maiores índices em relação aos outros setores do hospital, sendo o Índice de freqüência igual a 0,29 equivalente a $5,5 \%$ de tempo perdido.

JORGE (1995) constatou que a taxa de absenteísmo é de $4 \%$ entre trabalhadores de enfermagem de um hospital público.

MEIRA (1982), estudando a melhor forma de adaptação das taxas para o cálculo de absenteísmo na empresa na qual trabalhava, constatou a média da taxa de freqüência igual a $1,17 \%$, sendo até 15 dias igual a $1,10 \%$ e acima de 15 dias igual a $0,07 \%$, enquanto que a média do percentual igual a 3,09 , considerando \%Tp igual a 0,66 até 15 dias e $\%$ Tp igual a 2,42, estavam acima de
15 dias de ausência.

Segundo OTERO (1993), tem estudos sobre o absenteísmo hospitalar na Espanha, o índice de freqüência varia entre 0,2 a 0,8 seguindo a duração do tempo de afastamento, com uma distribuição do tipo logarítmico com uma moda de 1-2 dias, uma mediana de 8-9 dias e uma média em torno de 10-25 dias.

Segundo NOGUEIRA \& AZEVEDO (1982), estudos epidemiológicos bem conduzidos, permitem conhecer as diferentes variáveis que afetam o absenteísmo, como sexo, idade, estado civil, turnos de trabalho dias da semana, meses do ano, fatores psicológicos, causas médicas de faltas ao trabalho entre outros, assim como auxiliar na prevenção do mesmo.

Para FLIPPO (1973), os índices servem para destacar o problema, isto é, detectar as causas, e se forem usados com habilidade e prudência, pode ser efetuada uma medida de controle.

Se analisarmos isoladamente os valores encontrados para o índice de freqüência e porcentagem de tempo perdido, devemos afirmar que o absenteísmo está elevado entre os trabalhadores de enfermagem.

\section{CONCLUSÃO}

O absenteísmo foi entendido como as faltas do trabalhador de enfermagem às atividades laborais. Suas causas foram decorrentes de fatores como doença $(72,6 \%)$, acidentes do trabalho $(2,9 \%)$ e por motivos pessoais $(24,5 \%)$.

Os dados apontam que, entre os auxiliares de enfermagem, a Pediatria foi o local de trabalho onde se registrou maior Índice de freqüência (If $=0,35$ equivalente a 62 afastamentos), e o setor de Pronto Atendimento foi o local de trabalho onde se constatou maior número de dias perdidos $(\mathrm{T} p=4,19 \%$ equivalentes a 301 dias perdidos). Entre os enfermeiros, a UTI foi o local com maior Índice de freqüência, ou seja, If $=0,16$ e a maior Porcentagem de tempo perdido foi $4,14 \%$.

Concluímos que os índices de absenteísmodoença entre os trabalhadores de enfermagem da instituição estudada apresentam-se elevados, indicando a necessidade de estudos em cada local de trabalho, buscando detectar problemas causais específicos de cada setor e planejar soluções. Há também a necessidade de criação de um banco de dados para otimizar o registro das faltas, afim de facilitar seu controle e possibilitar futuras pesquisas. 


\section{ABSENTEEISM OF NURSING WORKERS FROM A UNIVERSITY HOSPITAL}

The purpose of this study was to identify the rates of absenteeism of nursing workers from a University Hospital due to diseases. Therefore, the author verified the number of absences due to health problems registered during 12 months, calculating the frequency rate as well as the percentage of wasted time. Data were collected and transcribed after consultation to reports organized by the human resources department of the institution studied. Results showed that the higher rate of absence occurred in the Pediatric Unit $(I f=0,35)$ and the higher percentage of wasted time occurred in the Emergency Unit (Tp=4,19). The author concluded that the rate of absenteeism due to disease was high among the workers, indicating the need of creating a database in order to optimize the registration of absences and their control as well as of stimulating the development of future research.

KEY WORDS: disease, absenteeism, nursing worker, nursing, clinical nursing human resources

\section{AUSENTISMO EN LOS TRABAJADORES DE ENFERMERÍA DE UN HOSPITAL UNIVERSITARIO}

Con el objetivo de identificar el indice de ausentismo/enfermedad entre los trabajadores de enfermería de un hospital universitario fue realizado un examen de las ausencias por incapacidad médica registrada durante doce meses, fue calculado el indice de frecuencia y el porcentaje de tiempo perdido. Los datos fueron recolectados a través de consulta documental en los registros del departamento de personal de la institución estudiada y copiados en un protocolo. De los resultados obtenidos, se constató que el mayor índice de frecuencia ocurrió en Pediatría $(I f=0,35)$ y el porcentaje de tiempo perdido de trabajo ocurrió en el servicio de Urgencias (Tp = 4,19). Se concluye que los índices de ausentismo/enfermedad entre los trabajadores se presentan elevados, indicando la necesidad de crear un banco de datos para optimizar el registro de faltas, facilitar el control y posibilitar futuras investigaciones.

TÉRMINOS CLAVES: ausentismo, enfermedad, trabajo, enfermería, hospital

\section{REFERÊNCIAS BIBLIOGRÁFICAS}

01. ALVES, A.R.A. Avaliação diagnostica dos índices de absenteísmo da equipe de Enfermagem de um Hospital de Ensino. Fortaleza, 1995. 74p.

02. ALEXANDRE, N.M.C. Avaliação de determinados aspectos ergonômicos no transporte de pacientes. Ribeirão Preto, 1987. Dissertação (Mestrado) - Escola de Enfermagem de Ribeirão Preto, Universidade de São Paulo.

03. BITTENCOURT, C.M. Doenças do trabalho e o exercício da enfermagem. Salvador, 1993. 96p. Dissertação (Mestrado) - Escola de Enfermagem, Universidade Federal da Bahia.

04. BULHÕES, I. Enfermagem do trabalho. Rio de Janeiro: IDEAS, 1986. v. 2, 463 p.

05. . Riscos do trabalho de enfermagem. 2 . ed. Rio de Janeiro: Correio Carioca, 1998. 221p.

06. CHIAVENATO, I. Recursos humanos. São Paulo: Atlas, 1985. 377p.

$07 . \quad$ Recursos humanos na empresa. 3. ed. São Paulo: Atlas, 1994. v. 2, 139p.

08. COUTO, H.A. Temas de saúde ocupacional: coletânea dos cadernos ERGO. Belo Horizonte: ERGO, 1987. 432p.

09. FERREIRA, A.B.de H. Novo dicionário da língua portuguesa, Rio de Janeiro, (s/n), 1986.1838p.

10. FLIPPO, E.B. Princípios de administração de pessoal. 2. ed. São Paulo: Atlas, 1973. v. 1.
11. GASPAR, P.J.S. Enfermagem profissão de risco e de desgaste: perspectivas do enfermeiro do serviço de urgência. Nursing - Ed. Portuguesa, v. 10, n. 109 , p. 24, mar. 1997.

12. JORGE, A.L. Motivos que levam os trabalhadores de enfermagem ao absenteísmo. Acta Paul. Enfermagem, São Paulo, v. 8, n. 1, p. 39-46,jan./ abr. 1995.

13. LOPES, G.T.; SPÍNDOLA, T.; MARTINS, E.R.C. O adoecer em enfermagem segundo seus profissionais: estudos preliminares. Rev. Enfermagem UERJ, v. 4, n. 1, p. 9-18, maio 1996.

14. MARZIALE, M.H.P. Condições ergonômicas da situação de trabalho, do pessoal de enfermagem, em uma unidade de internação hospitalar. Ribeirão Preto, 1995. 155p. Tese (Doutorado) - Escola de Enfermagem de Ribeirão Preto, Universidade de São Paulo.

15. MEIRA, J.B.B. Absenteísmo por enfermidade: sugestões para o seu controle. Rev. Bras. Saúde Ocup., v. 10, n. 40, p. 68-76,out./dez. 1982.

16. MOURA, G.M.S.S.de. O estudo da satisfação no trabalho e do clima organizacional como fatores contributivos para o ser saudável no trabalho da enfermagem. Texto Contexto Enfermagem, Florianópolis, v. 2, n. 1, p. 167-179, jul./dez. 1992. 
17. NAPOLEÃO, A.A. Causas de subnotificação de acidentes de trabalho: visão dos trabalhadores de enfermagem de um hospital do interior paulista. Ribeirão Preto, 1999.95 p. Dissertação (Mestrado) - Escola de Enfermagem de Ribeirão Preto, Universidade de São Paulo.

18. NOGUEIRA, D.P.; AZEVEDO, C.A.B. Absenteísmo - doença em mulheres. Rev. Bras. Saúde Ocup., v. 38, n. 10, p. $48-51,1982$.

19. OTERO, J.J.G. Riesgos del trabajo del personal sanitario. 2. ed. Madrid: McGRAW-HILL INTERAmericana de ESPAÑA, 1993.
20. QUICK, T.C.; LAPERTOSA, J.B. Análise do absenteísmo em Usina Siderúrgica. Rev. Bras. Saúde Ocup., v. 18, n. 69, p. 65-70, 1982.

21. REIS, I.N. Doenças ocupacionais: estudo retrospectivo em unidades hospitalares do DF. HFA - Publ. Téc. Cient., v. 1, n. 2, p. 113-122, jul./set. 1986.

22. ROBAZZI, M.L.C.C.; PARACCHINI, S.A. ; GIR, E.; SANTOS, W.D.F. dos; MORIYA, T.M. Serviço de enfermagem: um estudo sobre os absenteísmos. Rev. Bras. Saúde Ocup., v. 18, n. 69, p. 65-70, jan./fev./mar. 1990. 